\title{
THE ENDOCRINES IN THEORY AND PRACTICE
}

\author{
This article is one of a series on Endocrinology contributed by invitation
}

PITUITARY TUMOURS THEIR CLASSIFICATION AND TREATMENT

BY

\author{
J. H. BIGGART, M.D. \\ AND \\ N. M. DOTT, F.R.C.S.Ed.
}

From the clinical standpoint the term " pituitary tumours " is regarded as of regional rather than of histological significance. It embraces a variety of tumefactions of the pituitary region which cause symptoms indicative of glandular dysfunction, of chiasmal compression, and of hypothalamic derangement. For the purposes of differential diagnosis it is also necessary to include in our survey the regional effects of hydrocephalus. The regional group includes: (a) pituitary adenomas, (b) epidermoid pituitary tumours, (c) cysts of Rathke's pouch, $(d)$ miscellaneous tumours of the neighbourhood, and $(e)$ hydrocephalic distension of the third ventricle.

\section{Anatomical Basis}

There is a natural division into (a) tumours arising within the sella, and $(b)$ tumours arising above the sella. The division is significant in relation to symptoms and also in respect of surgical management.

Tumours arising within the sella include the adenomas, a proportion of epidermoid tumours, and the cysts of Rathke's pouch. The earliest symptom (except for the secreting eosinophil adenoma and perhaps the basophil adenoma) is hypopituitarism, expressed as infantilism in the young and sexual inactivation in the adult. $X$-ray examination of the sella shows a typical globular distension. Next, as the tumour enlarges upwards bitemporal hemianopia is found, and finally somnolence and other signs of hypothalamic damage may supervene. The tumour is in intimate contact with the dural lining of the sella and is usually adherent to it. The dura cannot be surgically removed because of its relation to the cavernous sinuses and carotid arteries. Hence, although the gross bulk of an intrasellar tumour mas be curetted away, it cannot be totally extirpated in a histological sense.

Tumours arising above the sella include a proportion of epidermoid tumours, rare adenomas from the pars tuber:lis, meningiomas of this region, and the other miscellaneous tumours mentioned. The chronology of symptoms varies according to the exact site of origin. The suprasellar epidermoid tumours are apt to show hypothalamic symptoms first-adiposity, polyuria, somnolence, etc.-then chiasmal bitemporal hemianopia; and, lastly, signs of glandular compression. The sella turcica is affected late and shows erosion of its outstanding clinoid processes rather than distension of its cavity. The meningiomas of more anterior origin usually evoke signs of chiasmal and optic nerve compression first, and these may be the only symptoms discovered. Most suprasellar tumours are susceptible of complete surgical removal.

In general, pituitary tumours differ from the majority of intracranial growths in that, while they produce a well-defined group of local signs, they do not give rise to signs of general intracranial hypertension. They do not cause papilloedema, but simple primary optic atrophy. Hydrocephalus simulating a pituitary tumour, on the other hand, is usually associated with signs of hypertension and with papilloedema. These features assist in the differentiation of primary tumours of the pituitary region from hydrocephalus simulating such tumours. A rare exception to the rule is the epidermoid pituitary cyst which enlarges into the third ventricle and blocks the foramina of Monro. It thus causes hydrocephalus and papilloedema and other symptoms of hypertension. The observation of a calcified nodule above the sella in the $x$-ray film may provide the clue to the diagnosis, but in the absence of this clue only ventriculography can distinguish these third ventricle tumours from hydrocephalus due to more distant causes.

\section{Endocrine Basis}

There is a natural division of pituitary tumours according as they (a) produce a hormone themselves, and (b) act merely as inert masses compressing the normal gland and thus suppressing the secretion of the normal gland. In other words, they may be classified as those exhibiting pathological hyperpituitarism and those causing hypopituitarism.

In the first group-exhibiting pathological hyperpituitarism-we certainly place the eosinophil adenoma with its associated syndrome of acromegaly or gigantism. When we see a patient with acromegaly or gigantism we know that he harbours an eosinophil pituitary adenoma. It is believed that the basophil adenoma with its associated syndrome of vascular hypertension, obesity, and virilism may also represent a type of pathological hyperpituitarism. The disease is associated with significant changes in the suprarenal cortex also, and its relationship to the basophil adenoma does not appear to be quite constant. Some doubt therefore remains as to what should be regarded as the primary lesion, and as to whether it is essentially an expression of pathological hyperpituitarism. In both eosinophil and basophil adenomas the hyperplasia concerns only one of the three secreting cell types of the normal anterior and posterior lobes of the gland. While the hormone of the cell type concerned is produced in excess, the tumour damages the normal gland and suppresses its secretion. Thus, although the associated clinical syndromes reflect a hypersecretion of one element, they may also portray the concomitant suppression of the hormones of the remainder of the gland. Lastly, we must take into account tumours and other lesions of the hypothalamus and brain stem which interfere with the nervous secretory control of the gland. That such lesions may cause a functional hyperpituitarism is evidenced by their association with pubertas praecox and other evidences of hyperpituitarism.

In the second group-exhibiting hypopituitarism-are the chromophobe adenomas, epidermoid tumours and all the other miscellaneous tumours of the neighbourhood, and hydrocephalus causing pressure on the gland. Again we must bear in mind that lesions interfering with the secretory nerve supply to the gland may give rise to total or fractional hypopituitarism. Thus tumours or other lesions of the hypothalamus may cause diabetes insipidus, dwarfism, or sexual dystrophy singly or in combination. 
It should be emphasized that with the rare exceptions of gliomas of the optic chiasm and of metastatic growths, tumours of the pituitary region are benign in character and hence offer a relatively favourable prognosis.

\section{Treaiment}

The available means of treatment for pituitary tumours are surgical removal and, in certain instances, $x$-radiation. The indication for surgical intervention is usually a pro-

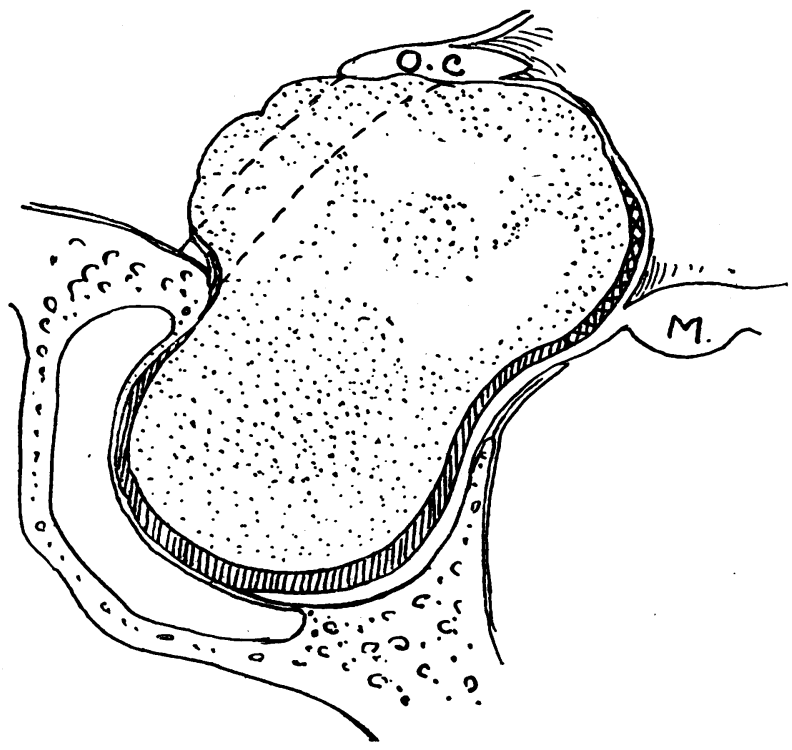

Fig. 1.-Typical intrasellar adenoma with upward extension. Note destruction of gland and compression of optic nerves and chiasm. O-C = optic chiasm. M $=$ mammillary body.

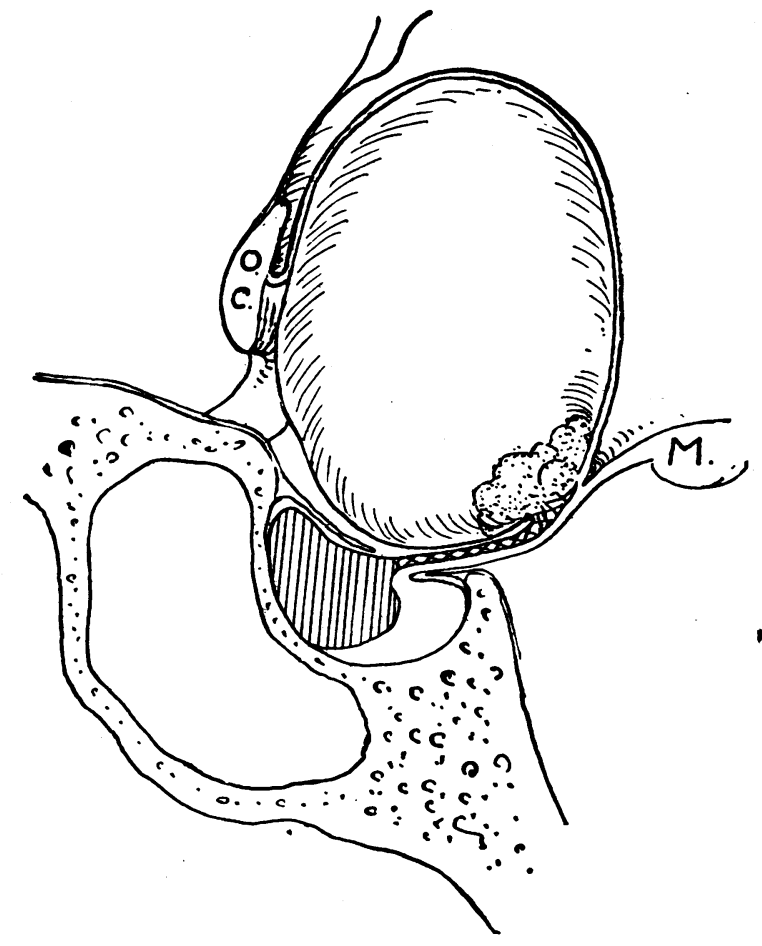

FIG. 2.-Typical suprasellar epidermoid cyst. Note hypothalamic distortion and secondary compression of optic chiasm and gland.

gressive impairment of vision from compression of the optic fibres at the chiasm, optic nerves, or tracts. These tumours rarely threaten life until very late in their course. Blindness is such a serious catastrophe, however, that the risks incidental to operation are fully justified in an attempt to avert it. Modern statistics indicate an operation risk to life varying from 5 to 10 per cent. Surgery is rarely resorted to in an attempt to combat endocrine disturbances. The most serious consequence of hyps. pituitarism in the adult is loss of sexual power, and removal of the tumour does not always restore gland function. Acromegaly, on the other hand, often runs a very slow and relatively benign course, hardly justifying the risks of operation in an attempt to stop its progress, unless vision is also threatened. In a few cases of rapidly progressive acromegaly, which may become very seriously disabling, surgical removal of the adenoma is probably justified in an attempt to arrest its progress.

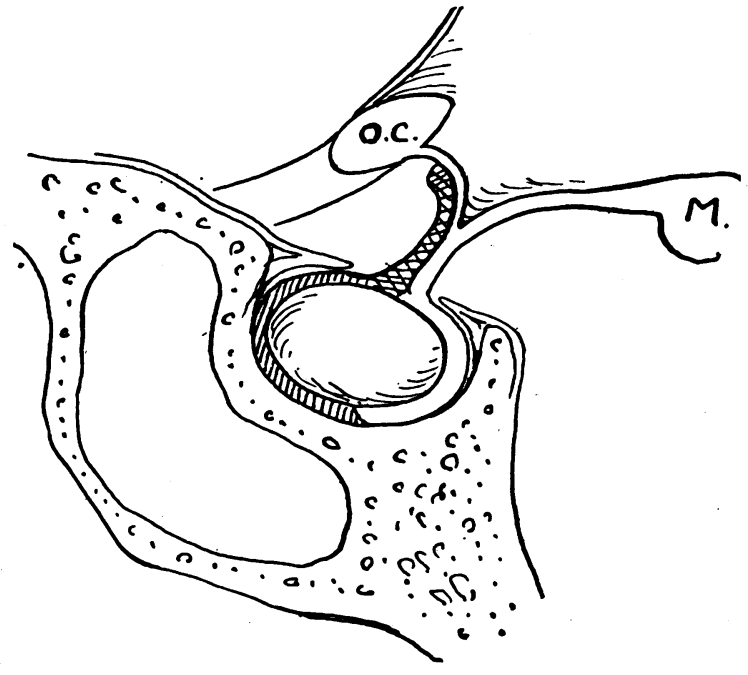

Fig. 3.-Rathke's pouch cyst. Note intraglandular situation and primary destruction of gland.

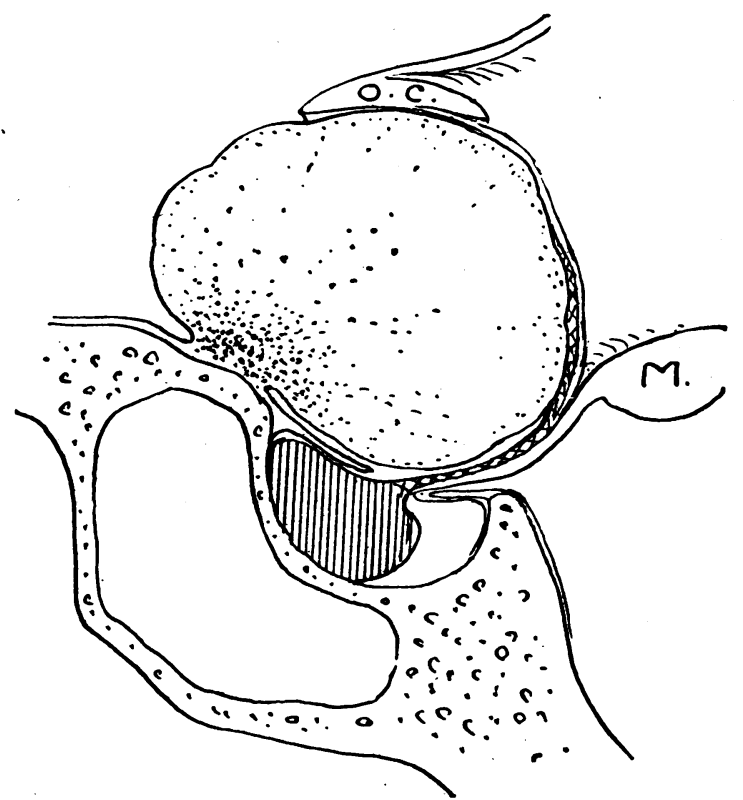

FIG. 4.-Meningioma of tuberculum sellae. Note primary distortion of optic nerves and chiasm and secondary compression of gland and hypothalamus.

\section{Operative Measures}

Most pituitary tumours are surgically exposed by reflecting an osteoplastic flap in the right or the left frontal region. The side with worse vision is usually selected, as it may be found advisable to sacrifice one optic nerve in order to secure better access and more complete removal of the tumour, thus adequately releasing and preserving the remaining nerve. The frontal lobe is gently lifted from the floor of the anterior fossa with a spatula until 
the optic nerves and chiasm and the tumour in relation to them come into view. In the case of a cyst, it is evacuated and its collapsed wall gently withdrawn through the interval between the optic nerves. In the case of a solid tumour, its interior is carefully excavated until its remaining outer shell can be collapsed and withdrawn in the same manner as in treating a cyst. In this way room to work cleanly under visual control and without damaging retraction of surrounding structures is gained. Great care is exercised in avoiding damage to the tuberal region of the base of the brain. If a tumour is actually adherent to this region, the adherent portion must be left undisturbed, for laceration of the floor of the third ventricle is usually fatal from the acute hypothalamic disturbances it gives rise to. One anticipates a complete removal of most of the meningiomas of this region and of a proportion of the epidermoid cysts. Adenomas, as already indicated, can never be quite completely removed, and, knowing this, the operator should err on the side of

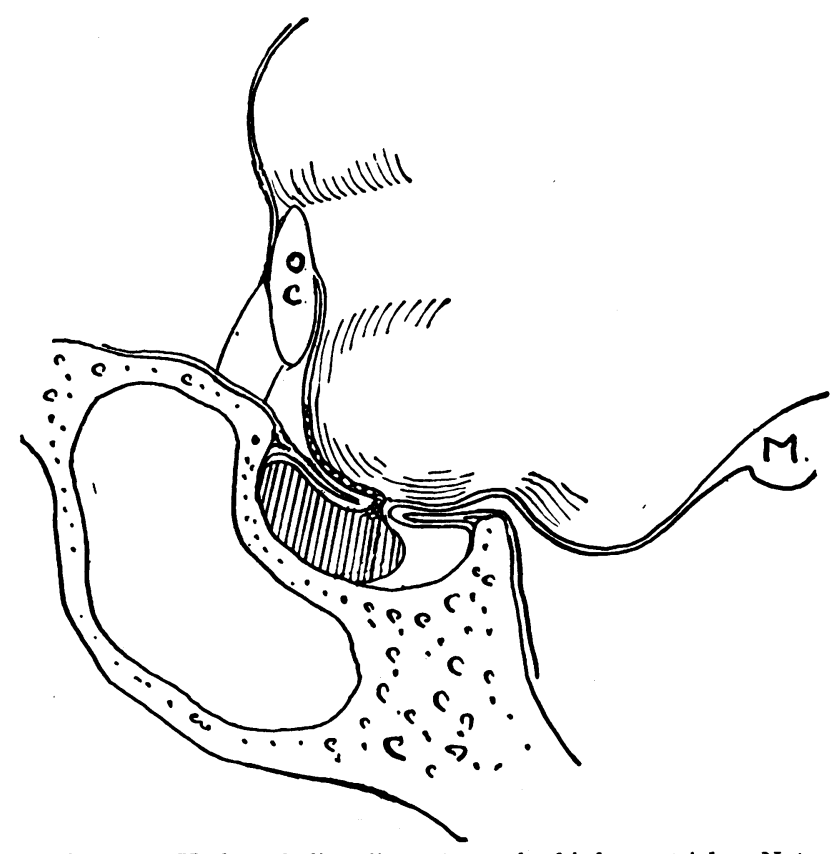

FIG. 5.-Hydocephalic distension of third ventricle. Note ballooning and thinning of the ventricular floor (hypothalamus) and secondary compression of gland and stretching of chiasm.

conservatism, and avoid carefully any risk of damage to surrounding structures, even at the expense of leaving a small proportion of the tumour untouched.

In some cases of obviously slowly-growing adenomas, especially those associated with acromegaly, the transsphenoidal operation gives excellent results. This procedure has fallen into rather undeserved neglect during recent years. The pendulum of progress has swung perhaps too far over to the intracranial procedure, as its technique has been improved and made safer. The transsphenoidal operation aims at removal of the bony floor of the sella turcica and subtotal removal of its contained adenoma. Since the tumour cannot be removed completely by any operation, and since the removal of the sellar floor permits any further growth of the remainder to enlarge harmlessly downwards into the sphenoidal cells, it provides very practical relief. This certainly is a less formidable operation than the intracranial procedure, and has fewer incidental risks. It is to bè recommended for slowly growing adenomas, especially in subjects who are regarded as poor surgical risks. It is, of course, only applicable to primarily intrasellar adenomas, and is not to be applied to any type of suprasellar tumour, nor to any case where doubt exists as to the nature of the lesion.

Rarely a pituitary tumour may be accessible only by a transventricular approach. This is true of the epidermoid cysts which come to occupy the third ventricle. The frontal lobe is incised, giving access to the anterior horn of the lateral ventricle. The tumour is seen through the foramen of Monro. In the case of a cyst it may be evacuated and withdrawn through the foramen. If more access is required the columns of the fornix are cut across. No obvious disability results from this procedure.

\section{Radiotherapy}

The place of radiotherapy in the management of pituitary tumours is at present undetermined. Although it has not been strikingly successful in the past recent improvements in technique give promise of better results. Radiotherapy has no obvious effect on tumours other than the adenomas. At the present time we employ radiotherapy regularly as a sequel and adjuvant to subtotal surgical removal. We do so in the hope of averting or postponing recurrence of the tumour. It takes many years to assess the value of such a procedure, and a firm opinion cannot be expressed upon it at present. We also employ radiotherapy in the primary treatment of adenomas in which visual impairment is absent or very slight. Results in the past year suggest that this treatment may suffice, but we are prepared to resort to surgery if their visual impairment should progress. Here again experience of modern radiotherapy is much too limited and too short to warrant an expression of opinion on its real value.

\section{Treatment of Cerfain Symptoms}

Lastly we may refer to the special treatment of certain symptoms of pituitary tumours. Most cases of diabetes insipidus can be greatly benefited by the administration of pituitrin or pitressin. According to the severity of the polyuria $1 / 2$ to $1 \mathrm{c} . \mathrm{cm}$. of pituitrin may be given hypodermically twice daily. In milder cases a single dose at night may suffice to avoid undue disturbance of sleep incidental to frequent urination. Pituitrin may also be administered by means of an intranasal spray or in the form of snuff. The results may be quite satisfactory, though they are less reliable than with the hypodermic method. A minority of cases of diabetes insipidus fail to benefit at all from pituitrin treatment. Recently it has been found that partial or total thyroidectomy greatly diminishes the polyuria of diabetes insipidus, and the operation has been performed for a few intractable cases with success.

Diabetes mellitus may complicate acromegaly. 'i he disease exhibits all the characters of true diabetes, and may end in diabetic coma. It may remain at a steady level for many months, or may show a conspicuous tendency to fluctuate. It reacts to dietetic and insulin treatment, just as true diabetes does, and should be treated on similar lines.

Sufferers from hypopituitarism may derive slight benefit from thyroid administration in rather small dosage. This tends to improve the symptoms which are attributable to their moderately depressed basal metabolic rate. At the present time no satisfactory pituitary substitution therapy is yet available to compensate for the major deficiencies of hypopituitarism-retardation of growth and sexual inactivation. One may assume, however, from the present state of knowledge of the hormones concerned, that their preparation in sufficient potency and purity for effective clinical administration is only a matter of time. 